\title{
Relationship between Early Menopause and Periodontal Disease in Korean Postmenopausal Women
}

\author{
Yun-Hee Lee, Sun-Mi Kim ${ }^{1}$, and Eunsuk $\mathrm{Ahn}^{\dagger}$ \\ Department of Dental Hygiene, Kyungbok University, Pocheon 11138, \\ ${ }^{1}$ Department of Dental Hygiene, Wonkwang Health Science University, Iksan 54536, Korea
}

\begin{abstract}
The purpose of this study was to evaluate the relationship between early menopause and periodontal disease in postmenopausal women using data from the 6th Korean National Health and Nutrition Examination Survey (2013 2015). A study was conducted with 2,048 postmenopausal women aged 45 to 74 years. Participants were divided into the early menopause group (menopause occurring at age 45 years or before) and normal menopause group (menopause occurring after age 45 years). A community periodontal index greater than or equal to code 3 was used to define periodontal treatment needs. A chi-square test was used to confirm the bivariate relationship between the variables. Multinomial logistic regression analysis was used to adjust for covariates (age, education, family income, body mass index, alcohol drinking, smoking, visiting dentist in the last year, use of oral care products, frequency of tooth brushing per day). The risk of periodontal disease was higher in the early menopause group after adjusting for potential confounders (odds ratio, 1.59). In particular, the relationship between early menopause and periodontal disease was more evident in women with low education and those who did not use oral care products. The findings of this study suggest that early menopause is a significant factor of periodontal disease in Korean women.
\end{abstract}

Key Words: Menopause, Periodontal diseases, Premature menopause

\section{Introduction}

Women have been found to experience periodontal disease related to sex hormones due to menstruation, pregnancy, and menopause as a result of physical changes over their lifetime ${ }^{1)}$. Changes in sex hormones are regarded as important factors that can affect the causes of periodontal disease ${ }^{2}$. Changes in estrogen, progesterone, and other female hormones have been reported to have an effect on interleukin-1 (IL-1), interleukin-6 (IL-6), and tumor necrosis factor- $\alpha$ (TNF- $\alpha$ ) secretion, which are proinflammatory cytokines playing a role in bone resorption and periodontal inflammation reactions ${ }^{3)}$. Moreover, since there are estrogen, progesterone, and androgen receptors in the gingiva, changes in female hormones have been reported to have a direct impact on the environment of the oral cavity ${ }^{4}$. Hormonal changes occur across a woman's lifetime, and some are related to menopause, a natural phenomenon where the menstrual cycle discontinues permanently owing to old age. The main cause of menopause is known to be decreases in estrogen and progesterone hormone levels resulting from atrophy of the ovaries ${ }^{5)}$. After menopause, xerostomia is a symptoms that can affect women's oral cavity). Estrogen maintains bone mass by accelerating bone production and decreasing bone resorption ${ }^{7}$, and its lack causes gum infections and leads to clinical attachment loss; thus, as the duration of menopause increases, clinical attachment loss and gingivitis also increase ${ }^{8)}$.

The age of menopause onset differs for each individual, 
and differences between races have also been identified; however, the average age of menopause is typically around the age of 50 years ${ }^{9)}$, and that of Korean women is 49.7 years. Given that women's life expectancy in Korea is 85.2 years as of 2015 , Korean women are expected to spend about 35.5 years or $1 / 3$ of their life in menopause ${ }^{10)}$. As a result of population aging, the number of women in menopause in the future will rapidly increase, which means the proportion of women in menopause in the entire population will also increase drastically. Middle-aged women in menopause typically have a stronger focus on their family than on themselves, and thus tend to neglect their own health. Due to a lack of knowledge regarding the various physical changes associated with menopause and their oral health needs, many women often fail to focus on their oral health ${ }^{11,12)}$. Therefore, improving awareness of the importance of managing oral health for women in menopause is extremely important from a health and social standpoint. While menopause starting around the age of 50 years is considered normal, early menopause starting before the age of 45 years is considered to be a morbid condition ${ }^{13)}$. According to previous studies, in primary early menopause (from natural causes) and secondary early menopause (resulting from surgery), bone density starts decreasing at an early age and the body is thus in a state of low bone density for an extended period of time, which increases the risk of osteoporosis and periodontal disease ${ }^{14,15)}$. Majority of the previous studies have either evaluated the risk of periodontal disease for women in menopause or analyzed the association between osteoporosis and early menopause. However, no studies to date have analyzed the association between early menopause and periodontal disease using representative data. Hence, this study aimed to verify the relationship between early menopause and the risk of periodontal disease among Korean women in menopause, and provide base data for establishing policies that can prevent periodontal disease and improve oral health for women in early menopause.

\section{Materials and Methods}

\section{Research participants}

Out of 4,503 women in menopause who participated in the 6th Korean National Health and Nutrition Examination Survey (2013 2015), after excluding those who experienced artificial menopause due to a hysterectomy, those with a doctor's diagnosis of diabetes with a strong relation to periodontal disease, and those with at least one response missing in the survey, a total of 2,048 participants were selected for analysis. Of these, 294 women whose menopause started at the age of 45 years or younger were assigned to the early menopause group, and 1,754 who started menopause after the age of 45 years constituted the normal menopause group. This study was conducted after receiving approval for exemption from the Institutional Review Board of Wonkwang University (WKIRB201803-SB-014).

\section{Variables}

In this study, the community periodontal index of treatment needs (CPITN), which ranges between 0 and 4 , was re-categorized into three levels, namely healthy periodontal tissue $(0)$, gingivitis $(1 \sim 2)$, and periodontitis $(3 \sim 4)$, and was used as the dependent variable. Menopause status (normal menopause group/early menopause group) was used as the independent variable. Moreover, this study also included participants' general characteristics, such as age group $(45 \sim 54 / 55 \sim 64 / 65 \sim$ 74 years), education level (elementary school or lower/ middle school/high school/college degree or higher), household income (lower/lower-middle/upper-middle/ upper), body mass index (BMI) (underweight/normal/ obese), lifestyle habits such as alcohol drinking (nondrinker/drinker in the past/current drinker) and smoking (non-smoker/smoker), and oral health management such visiting the dentist in the last year (yes/no), using oral management products (yes/no), and daily frequency of teeth brushing (less than 3 times a day/3 times a day or more).

\section{Analysis method}

Considering that the Korean National Health and Nutrition Examination Survey follows a complex sample design, a design file was generated prior to the analysis and the complex sample design was analyzed. The layer variable was the dispersed estimation layer, the cluster 
variable was survey district, the weighted values were the sample extraction framework, household at the point of the current survey, including error according to differences in population, and non-response error according to lack of participation in the survey. In order to adjust these factors, weighted values from a correlation analysis were used to calculate new weighted values. When cases are deleted, since complex sample information included in the deleted materials can be omitted and cause a standard deviation bias in the estimated values, the groups of interested and other groups were generated before the analysis. IBM SPSS statistics (ver. 22.0; IBM Co., Armonk, NY, USA) was used for all analyses. To assess participants' basic information, frequency analysis and descriptive statistics were performed. A chi-square test was also conducted to verify participants' menopause and periodontal statuses according to their sociodemographic characteristics, lifestyle habits, and oral health management status. Further, to assess the relationship between menopause status and periodontal disease, a multinomial logistic regression analysis was conducted to obtain the adjusted odds ratio (OR) and $95 \%$ confidence interval (CI). The statistical significance level was set to $\mathrm{p}<0.05$.

\section{Results}

1. Comparative analysis of general characteristics, lifestyle habits, and oral health management

Regarding the periodontal status of all 2,048 participants, $41.9 \%$ had gingivitis, $34.5 \%$ periodontitis, and $23.6 \%$ healthy periodontal tissue. As for education level, $35.6 \%$ were elementary school graduates or below, $32.1 \%$ high school graduates, $19.2 \%$ middle school graduates, and $13.1 \%$ college graduates or higher. Regarding household income level, 30.4\% were considered upper class, and $25.8 \%$ middle class. As for BMI, the majority of participants had a normal BMI (65.6\%). Regarding drinking and smoking, most participants were drinkers (58.3\%) and non-smokers $(93.7 \%)$, respectively. As for oral health management, $68.8 \%$ of participants reported not visiting a dentist in the last year, and $50.9 \%$ said they brushed their teeth at least 3 times a day (Table 1).

\section{Periodontal status according to general characteristics and menopause status \\ Upon examining periodontal status according to} menopause status, the number of early menopause with healthy periodontal tissue were more likely to be compared to the group with periodontitis, but this was not statistically significant. Women with periodontitis were more likely to be 65 to 74 years of age and elementary school graduates or below, compared to the group with healthy periodontal tissue $(\mathrm{p}<0.001)$. Moreover, women with periodontitis were more likely to have an average

Table 1. General Characteristics of the Study Population $(n=2,048)$

\begin{tabular}{|c|c|c|}
\hline Characteristic & Group & n (weighted \%) \\
\hline \multirow[t]{2}{*}{ Menopause status } & Normal menopause & $1,754(85.4)$ \\
\hline & Early menopause & $294(14.6)$ \\
\hline \multirow[t]{3}{*}{ Periodontal status ${ }^{\mathrm{a}}$} & Healthy & $472(23.6)$ \\
\hline & Gingivitis & 864 (41.9) \\
\hline & Periodontitis & $712(34.5)$ \\
\hline \multirow[t]{3}{*}{ Age (y) } & $45 \sim 54$ & $515(30.3)$ \\
\hline & $55 \sim 64$ & $976(48.1)$ \\
\hline & $65 \sim 74$ & $557(21.5)$ \\
\hline \multirow[t]{4}{*}{ Education } & Elementary and below & 799 (35.6) \\
\hline & Middle & 387 (19.2) \\
\hline & High & $593(32.1)$ \\
\hline & College and above & $269(13.1)$ \\
\hline \multirow[t]{4}{*}{ Family income $^{b}$} & Low & $436(19.3)$ \\
\hline & Middle low & $530(24.6)$ \\
\hline & Middle upper & $515(25.8)$ \\
\hline & Upper & $567(30.4)$ \\
\hline \multirow[t]{3}{*}{ Body mass index ${ }^{c}$} & Under & $41(2.0)$ \\
\hline & Normal & $1,315(65.6)$ \\
\hline & Over & $692(32.4)$ \\
\hline \multirow[t]{3}{*}{ Alcohol } & No & $460(20.2)$ \\
\hline & Past & $451(21.5)$ \\
\hline & Drinking & $1,137(58.3)$ \\
\hline \multirow[t]{2}{*}{ Smoking } & No & $1,926(93.7)$ \\
\hline & Yes & $122(6.3)$ \\
\hline \multirow[t]{2}{*}{ Dental visit $<1 \mathrm{y}$} & No & $1,417(68.8)$ \\
\hline & Yes & $631(31.2)$ \\
\hline \multirow{2}{*}{$\begin{array}{l}\text { Use of oral care } \\
\text { products }\end{array}$} & No & 975 (46.5) \\
\hline & Yes & $1,073(53.5)$ \\
\hline \multirow{2}{*}{$\begin{array}{l}\text { Frequency of tooth } \\
\text { brushing per day }\end{array}$} & $<3$ times & $1,044(49.1)$ \\
\hline & $\geq 3$ times & $1,004(50.9)$ \\
\hline
\end{tabular}

${ }^{a}$ Healthy, community periodontal index of treatment needs (CPITN) 0; gingivitis, CPITN 1 2; periodontitis, CPITN $3 \sim 4$. ${ }^{\mathrm{b}}$ Income quartile.

${ }^{c}$ Asia-Pacific Standard: under, $<18.5 \mathrm{~kg} / \mathrm{m}^{2}$; normal, $18.5 \sim$ $24.9 \mathrm{~kg} / \mathrm{m}^{2}$; obese, $\geq 25 \mathrm{~kg} / \mathrm{m}^{2}$. 
income level than those with healthy periodontal tissue ( $\mathrm{p}$ $<0.001$ ). Further, in the group with periodontitis, $38.6 \%$ of participants were obese, which was a higher proportion than among those with healthy periodontal tissue or gingivitis $(\mathrm{p}<0.001)$. Among those with healthy periodontal tissue, a higher percentage of participants used oral health products at $68.0 \%(\mathrm{p}<0.001)$ and visited a dentist within the last year at 38.3\% $(\mathrm{p}<0.01)$ (Table 2$)$.

\section{Impact of general characteristics and menopause status on periodontal disease}

Table 3 shows the results of the multinomial logistic regression analysis examining the effects of general characteristics and menopause status on periodontal disease. When all other factors were adjusted, the adjusted OR that a participant in the early menopause group would be afflicted by periodontitis compared to having normal menopause was 1.59 (95\% CI, 1.08 2.35; p<0.05). With respect to age, the adjusted OR that a woman between the age of 45 to 54 years would be afflicted by gingivitis was 1.80 (95\% CI, 1.18 2.75). Regarding education level, the adjusted OR that a woman who was an elementary school graduate or below would have gingivitis was 1.89 (95\% CI, $1.19 \sim 3.00 ; \mathrm{p}<0.01)$, and 2.09 for

Table 2. Periodontal Status Based on General Characteristics $(n=2,048)$

\begin{tabular}{|c|c|c|c|c|c|}
\hline \multirow[b]{2}{*}{ Characteristic } & \multirow[b]{2}{*}{ Group } & \multicolumn{3}{|c|}{ Periodontal status $^{\mathrm{a}}$} & \multirow[b]{2}{*}{ p-value } \\
\hline & & $\begin{array}{l}\text { Healthy } \\
(\mathrm{n}=472)\end{array}$ & $\begin{array}{l}\text { Gingivitis } \\
(\mathrm{n}=864)\end{array}$ & $\begin{array}{l}\text { Periodontitis } \\
(\mathrm{n}=712)\end{array}$ & \\
\hline \multirow[t]{2}{*}{ Menopause status } & Normal menopause & 399 (83.9) & $738(84.7)$ & $617(87.3)$ & 0.315 \\
\hline & Early menopause & $73(16.1)$ & $126(15.3)$ & $95(12.7)$ & \\
\hline \multirow[t]{3}{*}{ Age (y) } & $45 \sim 54$ & $119(29.7)$ & $266(36.7)$ & $130(23.0)$ & $0.000 * * *$ \\
\hline & $55 \sim 64$ & $253(54.9)$ & $383(44.0)$ & $340(48.5)$ & \\
\hline & $65 \sim 74$ & $100(15.3)$ & $215(19.2)$ & $242(28.6)$ & \\
\hline \multirow[t]{4}{*}{ Education } & Elementary and below & $127(23.2)$ & $339(35.4)$ & $333(44.3)$ & $0.000 * * *$ \\
\hline & Middle & $75(14.2)$ & $166(19.8)$ & $146(22.0)$ & \\
\hline & High & $179(43.7)$ & $257(32.7)$ & $157(23.4)$ & \\
\hline & College and above & $91(18.9)$ & $102(12.2)$ & $76(10.4)$ & \\
\hline \multirow[t]{4}{*}{ Family income $^{b}$} & Low & $76(13.6)$ & $191(19.6)$ & $169(22.8)$ & $0.000 * * *$ \\
\hline & Middle low & $109(20.4)$ & $227(25.1)$ & $194(26.7)$ & \\
\hline & Middle upper & $117(27.2)$ & $219(25.9)$ & $179(24.7)$ & \\
\hline & Upper & $170(38.9)$ & $227(29.3)$ & $170(25.8)$ & \\
\hline \multirow[t]{3}{*}{ Body mass index ${ }^{c}$} & Under & $17(3.2)$ & $13(1.5)$ & $11(1.7)$ & $0.000 * * *$ \\
\hline & Normal & $324(70.9)$ & $570(67.5)$ & $421(59.7)$ & \\
\hline & Over & $131(25.9)$ & $281(31.0)$ & $106(38.6)$ & \\
\hline \multirow[t]{3}{*}{ Alcohol } & No & $92(17.3)$ & 195 (19.9) & $173(22.6)$ & 0.281 \\
\hline & Past & $113(23.6)$ & 189 (21.9) & 149 (19.6) & \\
\hline & Drinking & $267(59.1)$ & $480(58.2)$ & $390(57.7)$ & \\
\hline \multirow[t]{2}{*}{ Smoking } & No & $452(95.0)$ & $814(93.8)$ & $660(92.6)$ & 0.392 \\
\hline & Yes & $20(5.0)$ & $50(6.2)$ & $52(7.4)$ & \\
\hline \multirow[t]{2}{*}{ Dental visit $<1$ y } & No & $284(61.7)$ & $637(72.1)$ & $496(69.8)$ & $0.004 * *$ \\
\hline & Yes & $188(38.3)$ & $227(27.9)$ & $216(30.2)$ & \\
\hline \multirow[t]{2}{*}{ Use of oral care products } & No & $160(32.0)$ & $426(47.6)$ & $389(55.2)$ & $0.000 * * *$ \\
\hline & Yes & $312(68.0)$ & $438(52.4)$ & $323(44.8)$ & \\
\hline \multirow[t]{2}{*}{ Frequency of tooth brushing per day } & $<3$ times & $218(44.2)$ & $450(50.1)$ & $376(51.1)$ & \\
\hline & $\geq 3$ times & $254(55.8)$ & $414(49.9)$ & $336(48.9)$ & \\
\hline
\end{tabular}

Values are presented as $\mathrm{n}$ (weighted $\%$ ).

$* * \mathrm{p}<0.01, * * * \mathrm{p}<0.001$

${ }^{\mathrm{a}}$ Healthy, community periodontal index of treatment needs (CPITN) 0 ; gingivitis, CPITN $1 \sim 2$; periodontitis, CPITN $3 \sim 4$.

${ }^{\mathrm{b}}$ Income quartile.

${ }^{\mathrm{c}}$ Asia-Pacific Standard: under, $<18.5 \mathrm{~kg} / \mathrm{m}^{2}$; normal, $18.5 \sim 24.9 \mathrm{~kg} / \mathrm{m}^{2}$; obese, $\geq 25 \mathrm{~kg} / \mathrm{m}^{2}$. 
Table 3. Periodontal Status Based on Menopause Status, General Characteristics, Lifestyle Habits, and Oral Care $(n=2,048)$

\begin{tabular}{|c|c|c|c|}
\hline \multirow{2}{*}{ Characteristic } & \multirow{2}{*}{ Group } & \multicolumn{2}{|c|}{ Periodontal status ${ }^{\mathrm{a}}$} \\
\hline & & Gingivitis & Periodontitis \\
\hline \multirow[t]{2}{*}{ Menopause status } & Early menopause & $1.27(0.87 \sim 1.84)$ & $1.59(1.08 \sim 2.35)^{*}$ \\
\hline & Normal menopause & 1.00 & 1.00 \\
\hline \multirow[t]{3}{*}{ Age (y) } & $45 \sim 54$ & $1.80(1.18 \sim 2.75)^{* *}$ & $0.80(0.51 \sim 1.24)$ \\
\hline & $55 \sim 64$ & $0.88(0.63 \sim 1.23)$ & $0.65(0.45 \sim 0.93)^{*}$ \\
\hline & $65 \sim 74$ & 1.00 & 1.00 \\
\hline \multirow[t]{4}{*}{ Education } & Elementary and below & $1.89(1.19 \sim 3.00)^{* *}$ & $2.09(1.29 \sim 3.40)^{*}$ \\
\hline & Middle & $1.84(1.12 \sim 3.02)^{*}$ & $2.25(1.38 \sim 3.67)^{*}$ \\
\hline & High & $0.99(0.67 \sim 1.46)$ & $0.83(0.55 \sim 1.26)$ \\
\hline & College and above & 1.00 & 1.00 \\
\hline \multirow[t]{4}{*}{ Family income $^{b}$} & Low & $1.37(0.91 \sim 2.07)$ & $1.24(0.79 \sim 1.94)$ \\
\hline & Middle low & $1.31(0.90 \sim 1.90)$ & $1.17(0.78 \sim 1.77)$ \\
\hline & Middle upper & $1.10(0.78 \sim 1.56)$ & $1.07(0.73 \sim 1.58)$ \\
\hline & Upper & 1.00 & 1.00 \\
\hline \multirow[t]{3}{*}{ Body mass index ${ }^{c}$} & Under & $0.47(0.21 \sim 1.06)$ & $0.49(0.20 \sim 1.21)$ \\
\hline & Normal & $0.94(0.69 \sim 1.27)$ & $0.76(0.56 \sim 1.02)$ \\
\hline & Over & 1.00 & 1.00 \\
\hline \multirow[t]{3}{*}{ Alcohol } & No & $1.25(0.89 \sim 1.75)$ & $1.28(0.90 \sim 1.80)$ \\
\hline & Past & $0.97(0.70 \sim 1.34)$ & $0.80(0.56 \sim 1.13)$ \\
\hline & Drinking & 1.00 & 1.00 \\
\hline \multirow[t]{2}{*}{ Smoking } & No & $0.80(0.42 \sim 1.51)$ & $0.57(0.29 \sim 1.13)$ \\
\hline & Yes & 1.00 & 1.00 \\
\hline \multirow[t]{2}{*}{ Dental visit $<1 \mathrm{y}$} & No & $1.30(0.96 \sim 1.76)$ & $1.01(0.74 \sim 1.38)$ \\
\hline & Yes & 1.00 & 1.00 \\
\hline \multirow[t]{2}{*}{ Use of oral care products } & No & $1.49(1.13 \sim 1.97)^{* *}$ & $1.89(1.40 \sim 2.56)^{* * *}$ \\
\hline & Yes & 1.00 & 1.00 \\
\hline \multirow[t]{2}{*}{ Frequency of tooth brushing per day } & $<3$ times & $1.06(0.81 \sim 1.39)$ & $0.97(0.71 \sim 1.32)$ \\
\hline & $\geq 3$ times & 1.00 & 1.00 \\
\hline
\end{tabular}

Values are presented as adjusted odds ratio (95\% confidence interval).

Adjusted for age (y), education, family income, body mass index, alcohol, smoking, dental visit $<1$ years, use of oral products, frequency of tooth brushing per day.

${ }^{*} \mathrm{p}<0.05, * * \mathrm{p}<0.01, * * * \mathrm{p}<0.001$

${ }^{a}$ Gingivitis, community periodontal index of treatment needs (CPITN) $1 \sim 2$; periodontitis, CPITN $3 \sim 4$.

${ }^{\mathrm{b}}$ Income quartile.

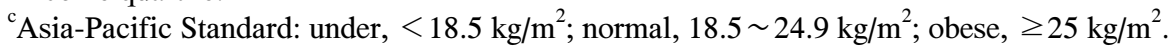

periodontitis (95\% CI, $1.29 \sim 3.40 ; \mathrm{p}<0.05)$, compared to having healthy periodontal tissue. For middle school graduates, the adjusted OR for having gingivitis compared to healthy periodontal tissue was $1.84(95 \% \mathrm{CI}, 1.12 \sim$ $3.02)$ and 2.25 for periodontitis $(95 \% \mathrm{CI}, 1.38 \sim 3.67$; p $<0.05)$. In other words, as education level decreased, the likelihood that a subject was afflicted by gingivitis or periodontitis increased. In terms use of oral health products, the adjusted OR that participants who did not use oral health products would be afflicted by gingivitis compared to having healthy periodontal tissue was 1.49 $(95 \% \mathrm{CI}, 1.13 \sim 1.97 ; \mathrm{p}<0.01)$ and 1.89 for periodontitis
(95\% CI, 1.40 2.56; $\mathrm{p}<0.001)$ (Table 3).

\section{Discussion}

This study used data from the Korean National Health and Nutrition Examination Survey in order to verify the effect of early and normal onset of menopause on periodontal diseases among pots-menopausal women. Here, periodontal disease was divided into gingivitis and periodontitis, based on previous studies reporting differences in bone density and attachment loss according to changes in hormones related to menopause ${ }^{7,16,17)}$, and we aimed to 
establish basic data that will be necessary for setting up policies for the prevention of periodontal disease and improvement of oral health for women in menopause.

The study results showed that women who experienced early menopause were at a higher risk of periodontitis compared to women undergoing normal-onset menopause. While a statistically significant association was clearly observed between early menopause and periodontitis, the association of early menopause with gingivitis was not as clear. According to the study conducted by Alves et al. ${ }^{8)}$, upon comparing the differences in menopause status and progress of periodontal disease, the depth of the paradental cyst was deeper in non-menopause women than in women in menopause; and Yalcin et al. ${ }^{18)}$ reported that periodontal disease was more common among women in menopause than non-menopause women, and that such disease appeared in more serious forms. These findings are consistent with the results of the present study. Further, when all other factors were controlled, the adjusted OR of periodontitis, which involves bone loss, was 1.59 in the group with early menopause compared to the group with normal menopause, which was statistically significant. Women with early menopause were also reported to have a higher risk of osteoporosis, cardiovascular diseases, and other relevant death risk factors compared to women with normal-onset menopause ${ }^{19)}$. Svejme et al. ${ }^{20)}$ reported that because bone remodeling is also affected by hormones, the lack of estrogen will have an impact on bone formation for patients with osteoporosis after menopause, which may lead to pathological bone remodeling and to periodontitis subsequently.

In addition, a study by the Women's Health Initiative divided women according to their menopause age into the under-40 group and 40-and-over group to compare bone density, and found that in the under-40 group, bone density was lower and the risk of fracture was higher ${ }^{21)}$. Because early menopause involves an extended period of estrogen deficiency, there is an increased risk for the early onset of osteoporosis and bone loss; therefore, there is a need for proper management to decrease and prevent periodontal disease among women experiencing early menopause. Moreover, it is important to increase awareness regarding these risks by providing training and information on the relationship of menopause with osteoporosis and oral health, and increasing access to information and local community resources for oral health behaviors from a preventative standpoint.

There was an association between the education level of participants and their risk of periodontal disease; the risk of gingivitis and periodontitis was about twice as high for women with a lower education level compared to those with a higher education level. Women's socioeconomic characteristics had an impact even when drinking, smoking, obesity, and other health-related variables were adjusted. These results are in agreement with previous studies that analyzed the relationship between women's health issues and socioeconomic factors. Women in a low socioeconomic position are a vulnerable health group ${ }^{22)}$; thus, more efforts must be made to resolve the issues leading to health inequalities. However, unlike education level, income level did not show a significant association with periodontal diseases, and this may be because this study used household income in the analysis, rather than participants' individual income level.

Rather than using clinical data, this study conducted an analysis based on the Korea National Health and Nutrition Examination Survey, which is reliable and representative on a national, city, and province level. However, no link was found between early menopause and periodontal diseases in this cross-sectional analysis. Since the start of the participants' menopause was before the starting point of the study, the status of confounding factors such as age, income may not be consistent, particularly if the gap between the two time point is long, and this may have been a limitation to this study. However, this research still holds significance in that it was the first study to provide basic data on postmenopausal women's health by revealing the factors related to early menopause and periodontal disease in a sample representative of Korean women; more research must be conducted on early menopause based on this study.

\section{References}

1. Suri V, Suri V: Menopause and oral health. J Midlife Health 5: 115-120, 2017. https://doi.org/10.4103/0976-7800.141187 
2. Im JS: The relationship between Korean women's premature menopause and osteoporosis: by using 2007, 2008 national health and nutrition examination survey. Unpublished master's thesis, Yonsei University, Seoul, 2012.

3. Jafri Z, Bhardwaj A, Sawai M, Sultan N: Influence of female sex hormones on periodontium: a case series. J Nat Sci Biol Med 6: 146-149, 2015. https://doi.org/10.4103/0976-9668.166124

4. Sharma N, Sharma RK, Tewari S, Chauhan M, Bhatia A: Association of periodontal inflammation, systemic inflammation, and duration of menopausal years in postmenopausal women. Quintessence Int 49: 123-131, 2018. https://doi.org/10.3290/j.qi.a39512

5. G K P, Arounassalame B: The quality of life during and after menopause among rural women. J Clin Diagn Res 7: 135-139, 2013. https://doi.org/10.7860/JCDR/2012/4910.2688

6. Chengappa R, Narayanan VS, Khan AM, Rakaraddi MP, Puttaswamy KA, Puttabuddi JH: Utility of two methodologies in the clinical assessment of oral dryness in postmenopausal women. J Midlife Health 7: 114-118, 2016. https://doi.org/10.4103/0976-7800.191014

7. Bijelic R, Milicevic S, Balaban J: Risk factors for osteoporosis in postmenopausal women. Med Arch (Sarajevo, Bosnia Herzegovina) 71: 25-28, 2017. https://doi.org/10.5455/medarh.2017.71.25-28

8. Alves RC, Félix SA, Rodriguez-Archilla A, Oliveira P, Brito J, Dos Santos JM: Relationship between menopause and periodontal disease: a cross-sectional study in a Portuguese population. Int J Clin Exp Med 8: 11412-11419, 2015.

9. Gold EB: The timing of the age at which natural menopause occurs. Obstet Gynecol Clin North Am 38: 425-440, 2011. https://doi.org/10.1016/j.ogc.2011.05.002

10. Korean Statistical Information Service: A life table in 2015. Retrived October 3, 2017, from http://meta.narastat.kr/ metasvc/svc/SvcMetaDcDtaPopup.do?orgId=350\&confmNo =920006\&kosisYn=Y (2016, December 2).

11. Park GJ, Park CH, Kim MO: Belief, knowledge, and practice about oral health care of middle-aged women. Korean $\mathrm{J}$ Women Health Nurs 15: 130-139, 2009. https://doi.org/10.4069/kjwhn.2009.15.2.130

12. Kwon HJ, Yoon MS: Relationship of depression, stress, and self-esteem with oral health-related quality of life of middle- aged women. J Dent Hyg Sci 15: 825-835, 2015.

13. Nelson LM: Primary ovarian insufficiency. N Engl J Med 360: 606-614, 2009.

14. Shuster LT, Rhodes DJ, Gostout BS, Grossardt BR, Rocca WA: Premature menopause or early menopause: long-term health consequences. Premature 65: 161-166, 2010. https://doi.org/10.1016/j.maturitas.2009.08.003

15. Tarkkila L, Furuholm J, Tiitinen A, Meurman JH: Oral health in perimenopausal and early postmenopausal women from baseline to 2 years of follow-up with reference to hormone replacement therapy. Clin Oral Investig 12: 271-277, 2008. https://doi.org/10.1007/s00784-008-0190-z

16. Yoshida N, Sugimoto K, Suzuki S, Kudo H: Change in oral health status associated with menopause in Japanese dental hygienists. Int J Dent Hyg 16: 157-164, 2018. https://doi.org/10.1111/idh.12282

17. Dutt P, Choudhary S, Kumar P: Oral health and menopause: a comprehensive review on current knowledge and associated dental management. Ann Med Health Sci Res 3: 320-323, 2013.

18. Yalcin F, Gurgan S, Gul G: Oral health in postmenopausal Turkish women. Oral Health Prev Dent 4: 227-233, 2006.

19. Svejme O, Ahlborg HG, Nilsson JÅ, Karlsson MK: Early menopause and risk of osteoporosis, fracture and mortality: a 34-year prospective observational study in 390 women. BJOG 119: 810-816, 2012. https://doi.org/10.1111/j.1471-0528.2012.03324.x

20. Svejme O, Ahlborg HG, Nilsson JA, Karlsson MK: Low $\mathrm{BMD}$ is an independent predictor of fracture and early menopause of mortality in post-menopausal women: a 34-year prospective study. Maturitas 74: 341-345, 2013. https://doi.org/10.1016/j.maturitas.2013.01.002

21. Sullivan SD, Lehman A, Nathan NK, Thomson CA, Howard $\mathrm{BV}$. Age of menopause and fracture risk in postmenopausal women randomized to calcium+vitamin $\mathrm{D}$, hormone therapy, or the combination: results from the Women's health initiative clinical trials. Menopause 24: 371-378, 2018. https://doi.org/10.1097/gme.0000000000000775

22. Kim YT, Ahn SS, Chung JJ, Choi SW: Korean women's health conditions and policy agenda. Korean Women's Development Institute, Seoul, 2008. 256 p. 